\title{
Improving Quality of Service in Cloud Computing
}

\author{
Mehreen Sirshar \\ Department of Software Engineering \\ Fatima Jinnah Women University \\ Rawalpindi, Pakistan \\ msirshar@gmail.com
}

Sobia Sajid

Department of Software Engineering

Fatima Jinnah Women University

Rawalpindi, Pakistan

sobiasajid97@gmail.com

\author{
Arooj Waheed \\ Department of Software Engineering \\ Fatima Jinnah Women University \\ Rawalpindi, Pakistan \\ aroojwaheed237@gmail.com
}

\author{
Sadia Ahmed \\ Department of Software Engineering \\ Fatima Jinnah Women University \\ Rawalpindi, Pakistan \\ sadia.366584@gmail.com
}

\begin{abstract}
Quality of Service (QoS) has a significant role in the provision of resources within service oriented distributed systems. In Quality of Service, cloud computing creates new challenges for improvements using the concept of virtualization. Currently, Cloud Computing is very emerging technology in every field of data storage and resource distribution over the network. Considering this new emerging technology, for the ease of data accessibility, price, resource use, restoration, response time and number of constraints the quality performance measures need to be upgraded. The paper highlights the research gap in providing a solution to achieve a Quality of Services in Cloud Computing. We also review the issues and challenges arising in cloud computing to guarantee quality.

Index Terms-Quality of Service; Cloud Computing; Virtualization; Data Accessibility; Challenges
\end{abstract}

\section{INTRODUCTION}

As cloud services are developing and there is fast development in cloud computing, many individuals and organizations are moving their work into the cloud. Considering cloud services to help cloud customers choose the most effective cloud service is a key subject. To achieve a high level of work, we need to safeguard the quality of the services provided to a client.

\section{ClOUd COMPUTING}

Cloud computing is the computing technology that preserves application and data using remote control servers and internet. It is an emergent technology. Cloud provides multi tenure, virtualization technology web services etc. resources over the internet. Cloud computing include three models. Software as a Service (SaaS) - It is an application that can be accessed from wherever at any time with an internet connection computer in hand. Infrastructure as a Service (IaaS) - Cloud service providers offers computer as physical or more frequently as virtual machines. Platform as a Service (PaaS) - Cloud service providers provides a pre-defined mix of operating system and application servers [1]. Quality of service also depends on the use of resources in different environment that can be characterized as:Private Cloud Computing - Virtualizing the resources used by a single organization and providing a virtual environment that can be hosted within or outside. Community Cloud Computing -
Virtual world services are distributed by various organizations in public cloud computing and are hosted outwardly. It can also be hosted internally by one organization. Public Cloud Computing In public cloud computing, infrastructure and resources are offered by organizations that also host the software for community use. Hybrid Cloud computing - This method of cloud computing comprises of two or more clouds that remain separate entities but are connected together, offering the benefits of multiple implementation models that can be shared both inside and outside [2].

\section{A. Characteristics of cloud services}

Different characteristics that make the cloud computing different from other computing systems includes Storage, cloud computing provides more storage than other memory storage devices as there is no worry of running out of space. Security, using cloud services means that the data is handed over to the third party and many users from different areas access the same server which results in security issues. Privacy, cloud computing offers password protection so that unauthorized users cannot access data. Flexibility, customers use cloud services from anywhere. Bring up-todate, it is the responsibility of cloud computing providers to provide updates and make them available, you just need to download and install them. Pricing of services, prices depends upon the demand of clients [3].

\section{B. Cloud computing architecture}

Cloud Computing architecture is the system layout of software components in cloud computing which includes the coordination of cloud components over a message line. Cloud computing architecture components includes the front-end platforms (mobile application), back-end platforms (server and services), and cloud based systems (internet etc.). The frontend and back-end are connected through network usually internet [4]. The figure below shows the cloud computing architecture. 

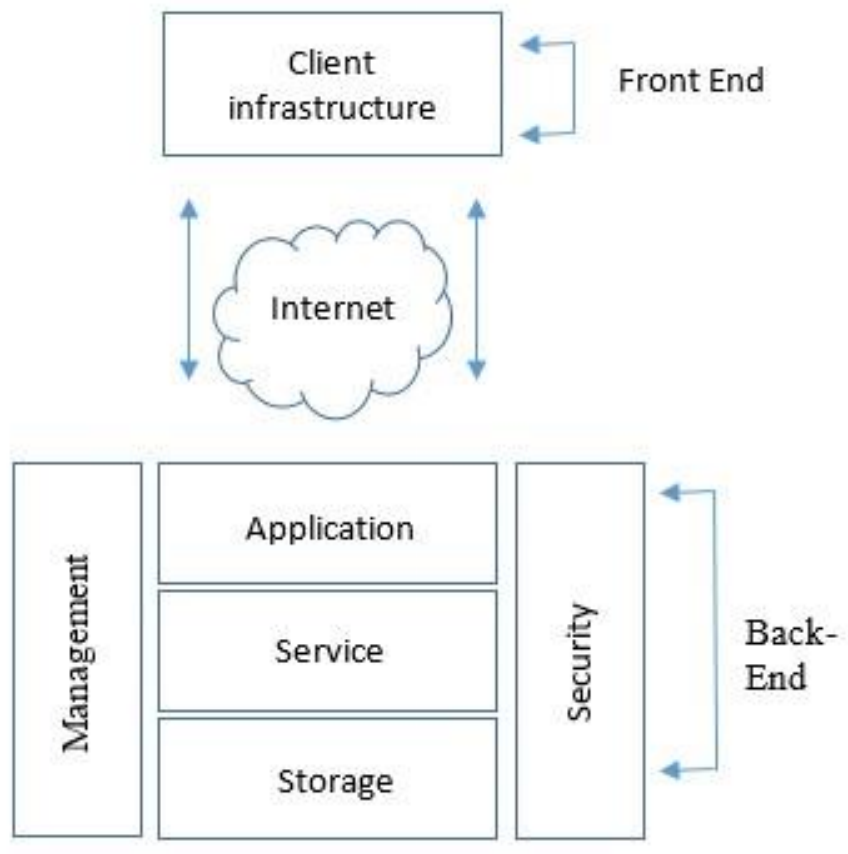

Fig. 1. Cloud Computing Architecture

\section{QUALITY OF SERVICE}

The term "Quality of Service" in Cloud Computing denotes the level of accessibility, dependability, security, usability and performance offered by the organization, platform and hosting applications. It is vital for the cloud customers who expect the cloud providers to distribute the quality features and cloud providers who need to find the right balance between the costs and service quality [5].

\section{A. Factors effecting the quality}

Quality is the totality of feature and characteristics of a product or service that bears its ability to satisfy the stated and implied needs. It is the measure of excellence or non-defect state, and significant variations [6]. There are many factors that affect the quality of systems.

Usability, the user interface of the software must be easy to user. Security, it is the important factor to ensure quality. Security policies like authentication and authorization must be implemented. Performance, it is the response time of the software. Robustness, software is robust if it is available even in the failure state. Platform compatibility, the quality software should run on as much platforms as it can so many users can use it. Maintainability, the system should be modifiable and able to manage the functionality without destroying the system to achieve quality.

\section{B. Techniques to improve Quality of service}

It is a challenge to implement quality of service in cloud computing. Different techniques to provide quality of service to cloud applications includes:

1) Resource allocation: The process of assigning accessible resources to the cloud application is dynamic resource provisioning. Allocation of resources will cause services to suffer if the allocation is not properly managed. Resource provisioning solves this problem by allowing individual module resource to be managed by service providers. Resource Allocation Strategy is about mixing service provider activities in order to assign inadequate resources within the cloud environment to meet the cloud application's needs [7].

2) Scheduling: Cloud computing scheduling is divided into two categories that is user level and system level. System level scheduling manages resources in data center. In data center, there are many physical machines. Scheduling of user request to physical machines is done in data center. Services given by cloud computing are based on Service Level Agreement, which is the contract between customer and service provider mentioning the terms of agreement including the nonfunctional requirement represented as quality of service [8].

3) Admission control: Admission control is to provide robust performance. The Infrastructure Provider should consider the extra requirement at the time of admission control along with the important computational and networking requirements that may need to be applied to run-time in order to become accessible [9]. 
C. Quality of Service (QoS) metrics

Fig. 2. Feature Metrics for Cloud Quality of Service

\begin{tabular}{|c|c|}
\hline Features & Metrics \\
\hline \multirow{3}{*}{$\begin{array}{c}\text { High Availability } \\
\text { and Fault } \\
\text { Tolerance }\end{array}$} & Flexibility \\
\hline & Response time \\
\hline & Recoverability \\
\hline \multirow{2}{*}{ Efficiency } & $\begin{array}{l}\text { Resource } \\
\text { Utilization }\end{array}$ \\
\hline & Delay and response time \\
\hline \multirow{2}{*}{ Usability } & Operability \\
\hline & User experience \\
\hline Modifiability & Mean Time To Change \\
\hline \multirow{2}{*}{$\begin{array}{l}\text { Portability and } \\
\text { scalability }\end{array}$} & Interoperability \\
\hline & Modularity \\
\hline
\end{tabular}

In cloud computing Quality of Services plays a key role in making the cloud services acceptable by the customers. Cloud computing can tie thousands of internationally distributed users at any time. QoS is the ability of any service to satisfy the stated and implied needs of the users. The metrics to use in Quality of Service depends on the functionality of the product. Metrics in term of performance, security, and economics are associated with cloud computing [10].

Performance metrics are relevant to the response time, timeliness and through put. The issues related to performance includes the response time required to process a demand, how much operations will be performed over a time period and the ability to process the requests in timely manner.

The economic properties includes price and elasticity. Due to different options available, economic metrics are used to compare and test different service costs. Metrics in term of elasticity are boot time, delete time, deployment time etc. For every business on the cloud, information security is important. The multi tenancy and virtualization concept is important for cloud security. Metrics such as communication and audit ability are required for the security of data. Some other metrics that are related to general features such as availability which include the metrics of flexibility and response time.

\section{ISSUES AND CHALLENGES}

There are major issues related with the management of cloud services. Cloud services growing trends makes it harder to examine the QoS for cloud. Although a lot of work have been done in the field of cloud computing service still there are some issues related to different parameters of Quality of Service.

The main concern is the security and confidentiality of data using cloud services. For every initiative, whether small or large, the security risks of cloud computing have become a reality. That's why a safe business intelligent cloud platform that can support appropriate security measures. The next challenge cloud computing facing today is of performance. When a client switches to cloud computing services, the cloud must provide improved services. Overall, performance is measured by the capabilities of cloud-based applications. Poor performance is due to lack of adequate resources. The next cloud computing risk involves cost. With the application requirement, the cost of virtual machine is dynamically changing. Less than needed virtual machines lead to a high use of resources. More Virtual Machines than necessary will result in resource waste. The required number standard is based on Quality of Service. Reliability and availability is also one of the major issue. One way to achieve reliability is to use redundant resources. Availability can be understood as the possibility of obtaining resources whenever necessary, taking into account the time it takes to provide the resources.

\section{CONCLUSION}

While emerging a software, quality of service is the important factor to consider. In the path of enlightening the quality of service in cloud computing, an analysis has been done. Many approaches and procedures are used to respond well to the constraints that play a dynamic role in improving cloud computing service quality. The parameters for the data stored on cloud computing over the network that must be enhanced are the response-time, price, and power-consumption.

\section{FUTURE WORK}

Future Work emphasis on increasing the rapid accessibility of data across the network to deliver maximum economical services and boost power-consumption, maximizing cloud computing service quality and other approaches to upturn Quality of Service parameters in cloud computing.

\section{REFERENCES}

[1] D. Puthal, B. Sahoo, S. Mishra and S. Swain, "Cloud Computing Features, Issues, and Challenges: A Big Picture," in International Conference on Computational Intelligence and Networks, Bhubaneshwar, India, 2015.

[2] D. Bermbach, "Quality of Cloud Services: Expect the Unexpected," 2017.

[3] F. F. Moghaddam, M. Ahmadi, S. Sarvani, M. Eslami and A. Golkar, "Computing Challenges and Opportunities: A Survey," in 1st 
Inrernational Conference on Telematic and Future Generation Networks (TAFGEN), Kuala Lumpur, Malaysia, 2015.

[4] "Cloud ComputingArchitecture"[Online].

Available: http://cloudcomputingnet.com/cloud-computingarchitecture/.

[5] D. D. K. Hashem H. Ramadan, "Quality of Service (QoS) in Cloud Computing," 2017.

[6] [Online]. http://www.businessdictionary.com/definition/quality.html.

[7] R. S. P. K. G. Shefali Varshney, "QoS Based Resource Provisioning in Cloud Computing Environment: A Technical Survey," 2019.

[8] A. I. ,. D. K. P. B. Abdallah Ali Zainelabden, "On Service Level Agreement Assurance in Cloud Computing Data Centers," 2016.

[9] P. B. R. H. H. K. Simranjit Kaur, "Quality of Service (QoS) Aware Workflow Scheduling (WFS) in Cloud Computing: A Systematic Review," 2018.

[10] S. P. Geeta, "A Review on Quality of Service in Cloud Computing," 2017.

[11] P. Manuel, "A trust model of cloud computing based on Quality of Service," 2015.

[12] D. N. J. I. G. A. E. B. K. Abdelzahir Abdelmaboud, "Quality of service approaches in cloud computing: A systematic mapping study," 2015.

[13] M. M. V. C. Mohamed Abdel-Basset, "NMCDA: A framework for evaluating cloud computing services," 2018.

[14] K. L. A. O. K. L. Jing Mei, "A Profit Maximization Scheme with Guaranteed Quality of Service in Cloud Computing," 2015.

[15] G.-Y. C. F.-F. C. Hassan Mahmood Khan, "An Adaptive Monitoring Framework for Ensuring Accountability and Quality of Services in Cloud Computing," 2016.

[16] A. K. D. M. A. R. M. A. M. M. H. A. A. Tamal Adhikary, "Quality of service aware cloud resource provisioning for social multimedia services and applications," 2016.

[17] E. M. V. S. D. V. Aleksandr Skatkov, "Visual Quality Estimation Technique for Services in Cloud Environments," 2017.

[18] M. Z. C. T. H. M. H. Ghahramani, "Toward Cloud Computing QoS Architecture: Analysis of Cloud Systems and Cloud Services," 2017.

[19] C. H. G. F. D. C. M. S. D. M. L. F. M. L. M. P. Bruno Guazzelli Batista, "A QoS-driven approach for cloud computing addressing attributes of performance and security," 2017.

[20] I. C. Sukhpal Singh, "QoS-aware Autonomic Cloud Computing for ICT," 2016.

[21] I. C. Sukhpal Singh, "Q-aware: Quality of service based cloud resource provisioning," 2015.

[22] S. P. N. S. C. Dhanamma Jagli, "SaaS CloudQual: A Quality Model for Evaluating Software as a Service on the Cloud Computing Environment," 2017.

[23] A. Sheshasaayee and . T. . S. Margaret, "SLA Based Utility Analysis for Improving QoS in Cloud Computin," in Advances in Intelligent Systems and Computing book series, 2016.

[24] S. Heidari and R. Buyya, "Quality of Service (QoS)-driven resource provisioning for large-scale graph processing in cloud computing environments: Graph Processing-as-a-Service (GPaaS)," 2019.

[25] P. Zhou, Z. Wang, W. Li and N. Jiang, "Quality Model of Cloud Service," in 17th International Conference on High Performance Computing and Communications (HPCC), 2015 IEEE 7th International Symposium on Cyberspace Safety and Security (CSS), and 2015 IEEE 12th International Conf on Embedded Software and Systems (ICESS), New York, NY, USA, 2015.

[26] A. A.-S. Ahmed and P. Andras, "Measuring the Scalability of CloudBased Software Services," in 2018 IEEE World Congress on Services (SERVICES), San Francisco, CA, USA, 2018.

[27] K. Bousselmi , Z. Brahmi and M. M. Gammoudi, "QoS-aware scheduling of Workows in Cloud Computing environments," in 2016 IEEE 30th International Conference on Advanced Information Networking and Applications, 2016.

[28] D. Ardagna, G. Casale, M. Ciavotta, J. F. Perez and W. Wang, "Qualityof-service in cloud computing: modeling techniques and their applications," Journal of Internet Services and Applications , 2014.
[29] S. Singh and I. Chana, "A Survey on Resource Scheduling in Cloud Computing: Issues and Challenges," Journal of Grid Computing, vol. 14, no. 2, 2016.

[30] A. Tchernykh, U. Schwiegelsohn, V. Alexandrov and E.-g. Talbi, "Towards Understanding Uncertainty in Cloud Computing Resource Provisioning," in ICCS 2015 International Conference On Computational Science, 2015. 